\title{
OUTCOME OF ST SEGMENT ELEVATED VERSUS NON- ELEVATED ACUTE MYOCARDIAL INFARCTION
}

Nazir A. Memon, Salma Kadir and Abdul Ghaffar Memon

\begin{abstract}
OBJECTIVE: To determine the outcome of patients with acute ST segment elevated myocardial infarction (STEMI) versus non -ST elevated myocardial infarction (NSTEMI) in our setup.

DESIGN: A descriptive study.

SETTING: Cardiology department, Liaquat University Hospital, Hyderabad - Sindh from $1^{\text {st }}$ May 2005 to $31^{\text {st }}$ July 2005.

METHODS: Out of $\mathbf{5 8 0}$ patients hospitalized for acute coronary syndrome, $\mathbf{4 2 8}$ patients of acute myocardial infarction were selected for the study. The patients were selected on the basis of raised biomarkers (CPK, CKMB, SGOT, and LDH/Trop-T) and one of the two i.e. electrocardiography (ECG) changes or history of chest pain. They were grouped into STEMI and NSTEMI.

RESULTS: Mean age of the patients was 52 years (range 35-75 years). Majority of patients (73\%) was male and $27 \%$ were females. Out of 428 patients selected for study, $288(67.28 \%)$ had STEMI while 140 (32.72\%) patients had NSTEMI. Recurrent chest pain was present in 85 (29.51\%) patients of STEMI; 45 (52.94\%) with ECG changes and 40 (47.05\%) without ECG changes. In STEMI group, complications were common, more in patients with recurrent chest pain and evidence of ECG changes as compared to those without ECG changes i.e. $16 \%$ and $10 \%$ respectively. Mortality was also higher (10.5\%) in patients of recurrent chest pain and ECG changes compared to those without ECG changes (6.8\%). Among 140 patients of NSTEMI, 48 (34.28\%) had recurrent chest pain; 30(62.5\%) with ECG changes and remaining 18(37.5\%) without ECG changes.

CONCLUSION: In NSTEMI and STEMI patients, recurrent chest pain and ECG changes are bad prognostic markers as compared to recurrent chest pain without ECG changes. These patients are more likely to suffer complications and can benefit from aggressive/invasive strategy than patients with recurrent chest pain without ECG changes. In NSTEMI group, complications and mortality are more frequent in patients with recurrent chest pain and ECG changes compared to those without ECG changes.
\end{abstract}

KEY WORDS: Myocardial infarction, Acute. Complications. Mortality.

\section{INTRODUCTION}

Coronary artery disease (CAD) is the leading cause of morbidity and mortality in the world. ${ }^{1}$ There is a high incidence of CAD in South Asia at an earlier age compared to other populations especially in the Western world. ${ }^{2,3}$ In Pakistan, in particular, its documented manifestations have been steadily increasing since early sixties. ${ }^{4-6}$ The manifestations of CAD include chronic stable angina, acute coronary syndrome (ACS), congestive heart failure, sudden cardiac death and silent ischaemia. ACS encompasses a spectrum of clinical conditions; from unstable angina to ST elevated myocardial infarction (STEMI) and non-ST elevated myocardial infarction (NSTEMI). The acute event usually represents a rupture of vulnerable atherosclerotic plaque, and platelet aggregation of varying degrees ensues, limiting blood flow to the myocardium distal to the lesion. The resulting supply demand mismatch can result in unstable angina, STEMI and NSTEMI. ${ }^{7}$ The development of STEMI is a fatal event in approximately one third of patients with about half of deaths occurring within one hour of the event from ventricular arrhythmia. ${ }^{8}$ The clinical diagnosis of $\mathrm{MI}$ requires an integrated assessment of the history with evidence of myocardial necrosis through biochemical markers, electro cardiographic and imaging modalities. Epidemiological reports from the World Health Organization and American Heart Association beginning in late 1950 required the presence of at least two of the following characteristic symptoms; 
ECG changes and a typical rise and fall in biochemical markers for the diagnosis of myocardial infarction. ${ }^{1}$ Intravenous thrombolytic therapy has led to a dramatic improvement in the outlook for patients with acute MI. However, reocclusion of the infarct- related artery occurs in some patients leading to increased morbidity and mortality. ${ }^{9}$ Timely reperfusion of jeopardized myocardium represents the most effective way of restoring the balance between myocardial oxygen supply and demand. When fibrinolysis is administered, the extent of protection appears to be related directly to the rapidity with which reperfusion is implemented after onset of coronary occlusion. ${ }^{10,11}$ Patients with STEMI and NSTEMI may develop complications. Most of them have recurrent chest pains and require readmission or prolonged hospital stay. This study was carried out to compare the outcomes of acute STEMI with NSTEMI with special reference to recurrent chest pain and mortality.

\section{PATIENTS AND METHODS}

This study was conducted from $1^{\text {st }}$ May 2005 to $31^{\text {st }}$ July 2005 in Cardiology department of Liaquat University Hospital, Hyderabad - Sindh. For this study following criteria were adopted.

\section{Inclusion criteria:}

\section{Biochemical markers:}

Raised cardiac enzymes like CPK + CKMB, SGOT and LDH within four hours of admission and other two sets 6 hours onward.

Positive Trop-T, 4 to 6 hours after the onset of symptoms.

The patients were selected on the basis of positive biomarkers as above and one of the following:

Electrocardiography: Either ST elevation of more than $1 \mathrm{~mm}$ in two consecutive leads or ST depression of more than $2 \mathrm{~mm}$ in two consecutive leads.

History: of chest pain of at least 30 minutes and within 48 hours.

These patients were also put in two groups:

(1) STEMI: ST elevation of $>1 \mathrm{~mm}$ in 2 consecutive leads with positive bio-chemical markers.

(2) NSTEMI: ST depression of $>2 \mathrm{~mm}$ in 2 consecutive leads with positive bio-chemical markers.

\section{Exclusion criteria:}

- Those patients having MI of more than 48 hours.

- If another cause of chest pain was established e.g. pericarditis or pneumonia.
Main outcome measures of the study were recurrent symptoms and mortality in both STEMI and NSTEMI groups. The patient's cardiovascular history, their risk factors (smoking status, history of hypertension, hyperlipidaemia, family history, diabetes, their inhospital clinical course including recurrent chest pain, maximum Killip class, and the initial diagnostic and treatment management were recorded on a predesigned proforma for each patient. Data was analyzed through SPSS version 10.0 software program. Equipment used for study purpose was three channel electrocardiography machine (Toshiba and Fakuda).

\section{RESULTS}

During study period, total 580 patients were hospitalized with acute coronary syndrome. However, 428 patients were studied as per eligibility criteria. Mean age of the patients was 52 years (range 35-75 years). Among these, $73 \%$ of the patients were male and 27\% female. Majority of the patients; 288 (67.3\%) was having STEMI and 140 (33.7\%) patients had NSTEMI. In the STEMI group, 180 patients (62.5\%) had anterior wall MI while 108 (37.5\%) patients inferior wall MI. Recurrent chest pain was observed in 85 (29.5\%) patients of STEMI; 45 patients (52.9\%) with ECG changes and 40(47\%) without ECG changes. In STEMI group, adverse events were more common in patients with recurrent chest pain and evidence of ECG changes as compared to those without ECG changes i.e. $16 \%$ and $10 \%$ respectively. Mortality was also higher (10.5\%) in patients of recurrent chest pain and ECG changes compared to those without ECG changes (6.8\%) (Table I). Among 140 patients of NSTEMI, 48 (34\%) had recurrent chest pain; 30 patients (62.5\%) with ECG changes and remaining 18 (37.5\%) without ECG changes. Similarly, NSTEMI group showed adverse events and mortality more frequently in patients with recurrent chest pain and ECG changes compared to those without ECG changes i.e. adverse events were $26.6 \%$ and $11 \%$ respectively while mortality was $13.3 \%$ and $10 \%$. Patients with NSTEMI were older, more often smokers and hypertensives, with frequent history of cardiovascular disease. Other risk factors and Killip class on admission, however, were not different for patients with or without STEMI (Table II). Total mortality in 428 patients was 42(9.8\%). In STEMI group, mortality was 25(8.6\%) among 288 cases while in NSTEMI 17 patients died (12.4\%) of 140 cases. 
TABLE I:

COMPLICATIONS IN STEMI / NSTEMI PATIENTS $(n=428)$

\begin{tabular}{|l|c|c|c|c|}
\hline \multicolumn{1}{|c|}{ Complication } & \multicolumn{2}{|c|}{ STEMI $(\mathbf{n = 2 8 8 )}$} & \multicolumn{2}{c|}{ NSTEMI $(\mathbf{n = 1 4 0 )}$} \\
\hline & $\begin{array}{c}\text { Recurrent Chest } \\
\text { pain + ECG } \\
\text { changes } \mathbf{n = 4 5}\end{array}$ & $\begin{array}{c}\text { Recurrent Chest } \\
\text { pain + No ECG } \\
\text { changes } \mathbf{n = 4 0}\end{array}$ & $\begin{array}{c}\text { Recurrent Chest } \\
\text { pain + ECG } \\
\text { changes } n=30\end{array}$ & $\begin{array}{c}\text { Recurrent Chest } \\
\text { pain + No ECG } \\
\text { changes } \mathbf{n = 1 8}\end{array}$ \\
\hline Arrythmias & $4(8.88 \%)$ & $4(10 \%)$ & $4(13.33 \%)$ & $1(5.55 \%)$ \\
\hline Heart failure & $1(2.22 \%)$ & 0 & $2(6.66 \%)$ & $1(5.55 \%)$ \\
\hline $\begin{array}{l}\text { Papillary } \\
\text { muscle dysfunction }\end{array}$ & $1(2.22 \%)$ & 0 & $2(6.66 \%)$ & 0 \\
\hline
\end{tabular}

TABLE II:

DISTRIBUTION OF RISK FACTORS IN STEMI I NSTEMI PATIENTS $(n=428)$

\begin{tabular}{|l|c|c|c|c|c|c|}
\hline \multicolumn{1}{|c|}{ Risk factor } & \multicolumn{3}{c|}{ STEMI (n= 288) } & \multicolumn{3}{c|}{ NSTEMI (n= 140) } \\
\hline & Total & Male & Female & Total & Male & Female \\
\hline Smoking & $143(49.65 \%)$ & $140(48.61 \%)$ & $3(1.4 \%)$ & $92(65.71 \%)$ & $90(64.28 \%)$ & $2(1.42 \%)$ \\
\hline Hypertension & $103(35.76 \%)$ & $78(27.08 \%)$ & $25(8.68 \%)$ & $59(42.24 \%)$ & $51(36.42 \%)$ & $8(5.71 \%)$ \\
\hline Diabetes & $95(32.98 \%)$ & $65(22.56 \%)$ & $30(10.41 \%)$ & $44(31.42 \%)$ & $38(27.14 \%)$ & $6(4.28 \%)$ \\
\hline Hyperlipidaemia & $75(26.04 \%)$ & $60(20.83 \%)$ & $15(5.20 \%)$ & $35(25 \%)$ & $30(21.4 \%)$ & $5(3.5 \%)$ \\
\hline Family history & $16(5.55 \%)$ & $10(3.47 \%)$ & $6(2.08 \%)$ & $11(7.85 \%)$ & $7(5 \%)$ & $4(2.85 \%)$ \\
\hline
\end{tabular}

\section{DISCUSSION}

Among 428 patients of acute myocardial infarction selected for this study, 288 (59.37\%) had acute STEMI, fulfilling the criteria of acute MI, and 140 (40.62\%) patients had NSTEMI. Among the patients of STEMI, acute anterior wall was involved in 71 (74.73\%) cases while in $24(25.26 \%)$ cases inferior wall was affected. Of 288 patients with STEMI, 85 (29.5\%) developed recurrent chest pain during their stay in hospital and out of these, 45 patients $(52.5 \%)$ had evidence of ischaemia like worsening of ECG changes and rising biomarkers. These patients actually qualified for early invasive strategy. In various studies from different parts of the world, there is a trend towards early invasive strategy and most of the guidelines show obvious benefit of early invasive workup. ${ }^{12,13}$ According to American Heart Association guidelines, patients with evidence of residual ischaemia (either by ECG, biomarker, or LV dysfunction) need early invasive treatment as there is upto $45 \%$ reduction of adverse events and 18\% reduction of mortality due to it. ${ }^{14}$ In this study, patients of STEMI group having recurrent chest pain with evidence of ECG changes were more likely to develop adverse events (i.e.16\%). Their mortality was also higher $(11.5 \%)$. In other words, ECG changes with chest pain predicted the trouble. Those patients with STEMI and recurrent chest pain but without ECG changes were less likely to suffer adverse events i.e. $10 \%$ and mortality of only $6.8 \%$. These patients need early conservative strategy and their pain should be evaluated on non-invasive workup. There is little data, comparing outcome of patients with STEMI and recurrent chest pain without residual ischaemia. GUSTO II study suggests that patients with ST elevation have higher mortality than NSTEMI. ${ }^{15}$ Although, ST depression had more adverse events, but in this study, overall adverse events in patients with STEMI were between $10 \%$ to $16 \%$ and mortality was $6.7 \%$ to $11 \%$, which is higher as compared to various other world centers, in agreement with the world literature but lower than the patients of NSTEMI in contrast to the world literature. This could be explained on the basis of public awareness; patient seeking and getting attention earlier shorter door to 
needle time, hence, myocardial salvage in the Western countries. The reverse is true for our population. In this study, out of 140 patients of NSTEMI group, 48(34\%) had recurrent chest pain while 30 patients $(62.5 \%)$ of this lot had evidence of residual ischaemia by persistent ST depression of $>2 \mathrm{~mm}$ and persistently raised biomarkers. They showed higher adverse event rate (26\%) and their mortality was $13 \%$. This is in agreement with different studies. ${ }^{16-18}$ Available literature suggests that these patients shall be referred for early invasive strategy. ${ }^{19,20}$ In TIMI TACTIS 18 study, there was $18 \%$ to $22 \%$ reduction of mortality in this group. ${ }^{21}$ However, in this study, it was found that not all recurrent chest pain patients were at high risk, but only those with evidence of ECG changes. Patients of NSTEMI who were without ischaemic evidence were having decreased adverse events and low mortality i.e. $11 \%$. This study shows higher mortality in patients with NSTEMI (12.4\%) as compared to available literature. ${ }^{16,18,20}$ Patients with NSTEMI comprise a growing subgroup of patients with ACS, producing a major public health problem worldwide, especially in Western countries, despite, significant improvements and refinements in management over the past 20 years. In United States alone, over 2.4 million people suffer from ACS each year, with more than six million individuals undergoing in-hospital vascular and cardiac surgery and related procedures. ${ }^{22}$ Consequently, much attention has been directed towards optimizing the diagnosis and management of such patients, particularly in light of the continued evolution of catheter-based interventions and newer pharmacologic strategies that affordmore complete platelet and thrombin inhibition. When used together, these approaches appear to have an important synergistic effect in reducing prognostically important ischaemic events. ${ }^{23,24}$ Recently, compelling evidence from randomized clinical trials has demonstrated that intermediate and high-risk ACS patients derive significant reductions in both morbidity and mortality with mechanical or surgical intervention, especially when revascularization is coupled with aggressive, multifaceted (antiplatelet, antithrombin, anti-ischaemic and antiatherogenic) medical therapy. ${ }^{20,24}$ For these reasons, it seems especially timely and appropriate to undertake a comprehensive review of the latest advances in the management of NSTEMI, mindful of the fact that even this noble effort to synthesize and integrate a prodigious amount of scientific information and cardiovascular therapeutics is destined to evolve still further as intensive efforts on optimizing clinical outcomes by harmonizing the advances in mechanical and pharmacologic interventions continue unabated.

\section{CONCLUSION}

This study concludes that STEMI/NSTEMI patients with recurrent chest pain and ECG changes are more likely to develop complications whereas patients with recurrent chest pain without ECG changes are least likely to suffer i.e. are a low risk group. Moreover, NSTEMI patients develop more complications than STEMI patients and benefit from early invasive strategy. This is a simple risk stratification, however, further improvement can be made by bringing in non invasive workup for further risk stratification.

\section{REFERENCES}

1. Newby LK, Rutsch WR, Califf RM, et al. Time from symptom onset to treatment and outcomes after thrombolytic therapy. Gusto-1 investigators. J Am Coll Cardiol. 1996;27:1646-55.

2. McKeigue PM. Coronary heart disease in Indians, Pakistanis and Bangladeshis: etiology and possibilities for prevention. $\mathrm{Br}$ Heart J. 1992; 67:341-42.

3. Fox KM. Heart diseases in Asians in Britain. $\mathrm{Br}$ Med J. 1998; 297:311-12.

4. Pirzada MA, Khan $\mathrm{AH}$. Coronary heart disease in Western Pakistan. Pak J Med Res. 1962;2:9-37.

5. Beg MA, Siddiqui MKJ, Abbasi AS, et al. Atherosclerosis in Karachi. J Pak Med Assoc. 1967; 17: 22.

6. Pakistan Medical Research Council. National Health Survey of Pakistan. Ministry of Health, Islamabad, 1998.

7. Alpert JS, Thygesen K, Antman E, et al. Myocardial infarction redefined: a consensus document of The Joint European Society of Cardiology/American College of Cardiology Committee for the redefinition of myocardial infarction. J Am Coll Cardiol. 2000; 36: 959.

8. Luepker RV, Apple FS, Christenson RH, et al. Case definitions for acute coronary disease in epidemiology and clinical research studies. Circulation. 2002; 108: 2543-55.

9. Katritsis D, Karvouni E, Webb-Peploe MM. Reperfusion in acute myocardial infarction: current concepts. Prog Cardiovascular Dis. 2003; 45: 481--93.

10. Rogers WJ, Canto JG, Lambrew CT, et al. Temporal trends in the treatment of over 1.5 million patients with myocardial infarction in the 
US from 1990 through 1999: The National Registry of MI 1, 2 and 3. J Am Coll Cardiol. 2000; 36: $2056-66$.

11. Kesteloot H, Sans S, Kromhout D. Evolution of all causes and cardiovascular mortality in the age group 75-84 years in Europe during the period 1970-1996: a comparison with worldwide changes. Eur Heart J. 2002; 23: 384--96.

12. Gurwitz JH, Goldberg RJ, Chen Z, et al. Recent trends in hospital mortality of AMI: The Worcester Heart Attack Study. Have improvements been realized for all age groups? Arch Intern Med. 1994; 154: 2202-8.

13. AHA Guidelines. Management of acute myocardial infarction. J Am Coll Cardiol. 2002; 44:671-719.

14. Armstrong PW, Fu Y, Chang WC, et al. Acute coronary syndrome in the GUSTO-IIb trial: prognostic insights and impact of recurrent ischaemia. Circulation. 1998;98:1860-8.

15. Stenestrand $U$, Wallentin L. Early revascularisation and 1-year survival in 14-day survivors of acute myocardial infarction: a prospective cohort study. Lancet. 2002; 359:1805-11.

16. Alter DA, Tu JV, Austin PC, et al. Waiting times, revascularization modality and outcomes after acute myocardial infarction at hospitals with and without on-site revascularization facilities in Canada. J Am Coll Cardiol. 2003; 42:410-19.

17. Gupta M, Chang WC, Van de Werf F, et al. ASSENT II Investigators. International differences in in-hospital revascularization and outcomes following acute myocardial infarction: a multilevel analysis of patients in ASSENT-2. Eur Heart $\mathrm{J}$. 2003; 24:1640-50.
18. Gibson CM, Karha J, Murphy SA, et al. TIMI Study Group. Early and long-term clinical outcomes associated with reinfarction following fibrinolytic administration in the thrombolysis in myocardial infarction trials. J Am Coll Cardiol. 2003;42:7-16.

19. Lablanche JM, Gueret $P$, Blanchard $D$, et al. Beneficial effects of very early PTCA in patients with intravenous thrombolysis for acute myocardial infarction: data from a nation-wide registry in France. Circulation. 2002; 106 (suppl II): 630 .

20. Cannon CP. Small molecule glycoprotein IIb/IIla receptor inhibitors as upstream therapy in acute coronary syndrome: insights from the TACTICS TIMI-18 trial. J Am Coll Cardiol. 2003; 41:43S$48 \mathrm{~S}$.

21. American Heart Association. 2002 Heart and Stroke Statistical Update. Dallas, Texas: American Heart Association; 2001.

22. Invasive compared with non-invasive treatment in unstable coronary-artery disease: FRISC II prospective randomised multicentre study. FRagmin and Fast Revascularisation during InStability in Coronary artery disease Investigators. Lancet. 1999;354:708-15.

23. Herrmann HC, Moliterno DJ, Ohman EM, et al. Facilitation of early percutaneous coronary intervention after reteplase with or without abciximab in acute myocardial infarction: results from the SPEED (GUSTO-4 PILOT) trial. J Am Coll Cardiol. 2000;36:1489-96.

24. Montalescot G, Barragan P, Wittenberg $O$, et al. Clinical benefits of platelet glycoprotein IIb/IIla blockade with coronary stenting in acute myocardial infarction. $N$ Engl $J$ Med. 2001;344:1895-1903.

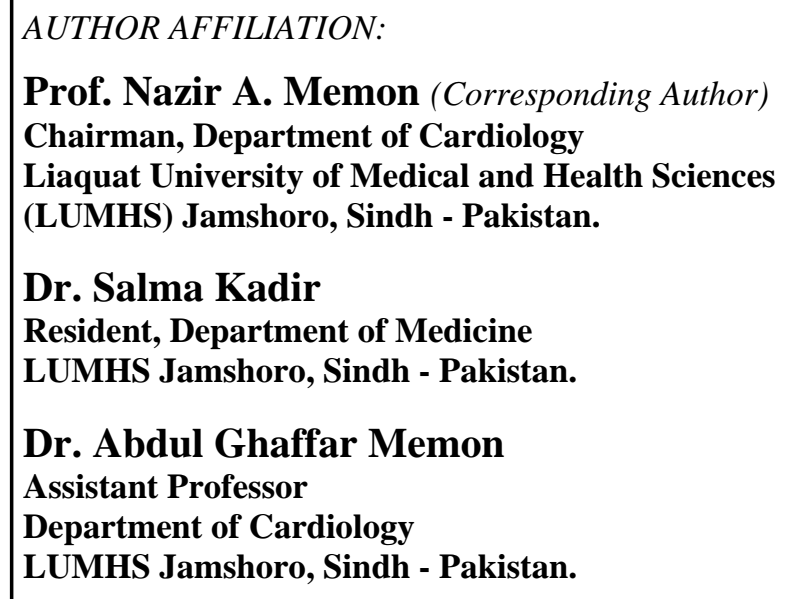

